\title{
Agôn
}

Revue des arts de la scène

7| 2015

La Distribution

\section{Distributions et inégalités de valorisation dans le spectacle vivant}

\section{Daniel Urrutiaguer}

\section{(2) OpenEdition}

\section{Journals}

\section{Édition électronique}

URL : http://journals.openedition.org/agon/3205

DOI : 10.4000/agon.3205

ISSN : 1961-8581

Éditeur

Association Agôn

\section{Référence électronique}

Daniel Urrutiaguer, « Distributions et inégalités de valorisation dans le spectacle vivant », Agôn [En ligne], 7 | 2015, mis en ligne le 13 juillet 2015, consulté le 03 mai 2019. URL : http:// journals.openedition.org/agon/3205; DOI : 10.4000/agon.3205

Ce document a été généré automatiquement le 3 mai 2019.

Association Agôn et les auteurs des articles 


\title{
Distributions et inégalités de valorisation dans le spectacle vivant
}

\author{
Daniel Urrutiaguer
}

1 Le terme de distribution peut avoir d'autres sens que la répartition des artistes interprètes dans les rôles des pièces ou des films et des professionnels technicoartistiques impliqués dans le montage et l'exploitation des spectacles. En économie, cette notion désigne usuellement l'ensemble des opérations qui permettent de mettre les produits à la disposition des consommateurs. Pour la filière des arts du spectacle, la distribution concerne la mise en connexion des producteurs et des lieux de diffusion qui programment les spectacles. Enfin en statistique, le terme est couramment employé pour désigner la répartition des valeurs prises par une variable. L'objet de l'article est d'apporter des éclairages socioéconomiques pour comprendre les logiques des choix de distribution dans un secteur où les inégalités de revenu sont plus élevées que pour la moyenne des salariés et où la figure des intermittents prédomine pour le montage et l'exploitation des spectacles.

2 En effet, la Comédie-Française est la seule troupe théâtrale permanente, recrutée selon un système de cooptation hiérarchisé. Elle comprend actuellement 61 sociétaires et pensionnaires alors que le recensement de la population active par l'INSEE comptait 30100 artistes dramatiques en $2010^{1}$. Le nombre de danseurs permanents dans les ballets était estimé à 500 salariés contre 4300 intermittents selon les données de la Caisse des Congés du Spectacle en $2000^{2}$. Selon les mêmes sources, les orchestres permanents comptaient 2000 musiciens tandis que 26000 intermittents étaient recensés en $2003^{3}$.

3 Cette domination des intermittents du spectacle parmi les artistes interprètes s'est accompagnée d'une forte croissance démographique de ces professions depuis les années 1990. Entre 1991 et 2011, le nombre d'artistes des spectacles a progressé de $66 \%$, celui des professionnels technico-artistiques des spectacles a été multiplié par 2,1 tandis que l'ensemble de la population active occupée n'a augmenté en France que de $16 \%^{4}$. Cette hausse a été la plus sensible dans les années $1990^{5}$. Parallèlement, cette expansion a été suivie d'un développement important de la flexibilité des relations d'emplois artistiques, les trois-quarts des artistes des spectacles salariés ayant des contrats courts et la moitié 
travaillant à temps partiel en 2011 alors que cela n'est le cas respectivement que de $40 \%$ et $15 \%$ des professionnels technico-artistiques des spectacles, $13 \%$ et $19 \%$ de l'ensemble des actifs occupés en $2011^{6}$. De plus, les inégalités de rémunération sont particulièrement accentuées selon les degrés de réputation corporative et de notoriété des artistes et des collaborateurs technico-artistiques, qui influent sur la distribution des salaires journaliers et des volumes d'engagement. La part des travailleurs pauvres est importante. Ainsi, en 2005 , les $25 \%$ des salariés les plus faiblement rémunérés se sont partagés $1 \%$ du revenu disponible total et les $10 \%$ les mieux payés $32 \%$ du revenu disponible total dans le spectacle vivant contre $5 \%$ et $32 \%$ respectivement pour l'ensemble du secteur privé7.

4 La construction d'une distribution artistique et technique s'effectue ainsi le plus fréquemment dans le cadre d'un projet de spectacle spécifique porté par un metteur en scène ou un chorégraphe avec des collaborateurs recrutés sur des durées limitées au montage et à la première exploitation du spectacle. Le nomadisme des interprètes s'accentue avec la réduction de la durée des contrats ${ }^{8}$ dans un contexte où l'accès au régime d'assurance chômage des intermittents du spectacle procure en moyenne un revenu de complément équivalent au salaire pour les artistes ${ }^{9}$. Le cumul des fonctions de direction artistique et d'interprétation est plus fréquent dans le spectacle vivant que dans l'audiovisuel. Ainsi, $32 \%$ des comédiens ont déclaré en 1994 avoir exercé aussi une fonction de mise en scène ${ }^{10}$ tandis que cela a été le cas de $44 \%$ des danseurs pour le rôle de chorégraphe en $2000^{11}$. De nombreux artistes alternent ainsi selon les projets des positions d'interprète et de directeur artistique, responsable de la sélection d'une distribution pour la création du spectacle. Le sentiment de croiser les statuts de salarié et d'employeur était partagé par un tiers des intermittents interrogés par l'enquête "d'initiative citoyenne » menée en 2003 par le laboratoire Ysis-Matisse de Paris 1. Cette hybridation ressentie de statuts s'accroît avec l'âge et se réduit quand la rémunération du travail artistique et le volume horaire des contrats de travail progressent ${ }^{12}$.

5 L'objet de cet article est d'abord de mettre en perspective par une approche conventionnaliste ${ }^{13}$ les explications économiques de ces inégalités de revenus du travail artistique. Ces explications se réfèrent à deux visions différentes pour expliquer la concentration de la demande sur les vedettes: les effets des différences de talent, considéré comme une capacité remarquable objectivement observable, et les effets d'auto-renforcement des notoriétés des artistes, par le bouche-à-oreille et les médias. L'approche théorique sur les inégalités de valorisation du travail artistique sera complétée par une analyse des interactions entre la pluralité des logiques de production et les choix de distribution artistique et technico-artistique. Une dernière partie abordera plus spécifiquement les résultats d'une enquête sur les territoires et les ressources des compagnies en France afin de s'interroger sur les effets du durcissement des rapports de diffusion entre les compagnies et les établissements culturels.

\section{Explications économiques des inégalités de revenu artistique : entre positivisme et constructionnisme}

\section{Les modélisations positivistes du talent}

$\mathrm{Au} \mathrm{XVIII}^{\mathrm{e}}$ siècle, dans son ouvrage De la richesse des nations, l'économiste Adam Smith a dégagé une cause des inégalités de salaires dans les différences d'éducation, « plus longue et dispendieuse dans les arts qui demandent une grande habileté ${ }^{14}$. Sans se référer 
nécessairement au processus éducatif comme Smith, l'économiste Alfred Marshall a mis en avant à la fin du XIX ${ }^{e}$ siècle la rareté des "capacités naturelles extraordinaires " comme la source d'une rente ${ }^{15}$. La vision de la rareté pour expliquer les inégalités de rémunération est reprise au début des années 1980 par l'économiste américain Sherwin Rosen dans la construction de son modèle sur les revenus des « superstars »"

Dans ce modèle centré sur des marchés cloisonnés de services très qualifiés (sports, chirurgie, services juridiques, arts par exemple), ce sont les différences de talent, entendu comme les capacités naturelles des personnes, qui déterminent la qualité des services offerts. L'hypothèse essentialiste du talent comme un état objectivement observable et connu de tous revient à considérer que les consommateurs ne sont différenciés que par leurs revenus. Dans ces conditions, la demande adressée aux professionnels pour un service donné suit une croissance exponentielle à mesure que leur talent s'affine. La satisfaction retirée de la production d'un artiste plus talentueux est ainsi supposée supérieure, ce qui attire le consentement à payer d'un plus grand nombre de consommateurs. Ce phénomène d'amplification des inégalités de revenus à partir de petits écarts de talent est accentué pour les marchés plus étendus comme dans le cas des industries culturelles. Glenn Mac Donald a proposé en 1988 un modèle complémentaire afin de lever l'hypothèse irrecevable de l'observation du talent sans coût d'information. Deux générations d'artistes, débutants et confirmés, sont différenciées. Le modèle suppose que le talent d'un artiste ne se modifie pas au cours de sa carrière et que le repérage de ses capacités est effectué de façon neutre par les journalistes culturels. Ceuxci révèlent ainsi de façon consensuelle les informations qui déterminent le maintien ou non sur le marché des artistes débutants par la suite ${ }^{17}$. Cette hypothèse est tout aussi peu convaincante; elle réduit la fonction des critiques dramatiques, chorégraphiques ou musicaux à des constats objectifs sur la "qualité » des représentations et est décalée quant au contraste entre la croissance démographique des artistes des spectacles et la réduction de la place occupée par les rubriques des critiques d'art dans la presse et les médias audiovisuels.

Le modèle de Mac Donald à deux générations imbriquées a été testé par William Hamlen en 1994 en s'appuyant sur les deux segments du marché industriel de la musique populaire, celui des disques en 45 tours, format des débuts, et celui des 33 tours réservé aux artistes confirmés ${ }^{18}$. L'échantillon a concerné 115 chanteurs entre 1955 et 1987 avec différents niveaux de ventes, depuis les artistes n'ayant jamais figuré dans le Top 1000 jusqu'à des stars comme Elvis Presley ou les Beatles. La mesure objective du talent proposée est celle du contenu de la voix en harmoniques aigus auxquels sont particulièrement sensibles les mélomanes. Il se réfère ainsi au mode de réception des experts, et non pas de l'ensemble des consommateurs, en leur conférant implicitement un rôle de juges éclairés, comme dans le modèle des normes de goût de David Hume ${ }^{19}$. Contrairement à la prédiction du modèle de Rosen sur l'amplification des inégalités de rémunération par les différences de talent artistique, les résultats du test économétrique indiquent une corrélation positive mais faible de cette variable du talent avec les revenus des chanteurs. Des variables explicatives autres que la tessiture de la voix ont été testées dans le modèle économétrique. Hamlen a dégagé une corrélation nettement plus significative pour des variables sociétales. Cette corrélation est négative pour les chanteurs noirs, positive pour les femmes et positive aussi pour des indicateurs de notoriété comme la composition antérieure de musiques de films, l'appui sur un orchestre ayant une bonne image, l'ancienneté de l'édition du premier disque. La mesure 
proposée du talent est toutefois fort réductrice et ne prend pas notamment en compte d'autres capacités comme celles de se mettre en scène lors des concerts, des clips ou lors des entretiens dans les médias.

\section{Les visions constructionnistes de la notoriété}

9 Sans se référer à une mesure du talent, un autre type de modélisation économique se focalise sur les effets d'imitation dans les choix de consommation culturelle afin de réduire les coûts liés au temps de recherche des informations sur la qualité des spectacles, par ailleurs incertaine car elle est liée à l'expérience subjective de la représentation. Cela augmente la probabilité de pouvoir échanger avec les autres sur les spectacles associant des vedettes puisqu'ils sont vus par le plus grand nombre. En se limitant aux coûts temporels de la consommation artistique à minimiser, celui de la recherche d'informations et celui de l'assistance aux représentations, et en supposant l'existence de segments de marchés avec des consommateurs aux préférences similaires, Moshe Adler a proposé en 1985 une modélisation aléatoire des inégalités de revenus artistiques qui ne dépend pas des différences de talent ${ }^{20}$. La minimisation des coûts d'information incite à imiter les choix des autres; des artistes ayant le même niveau de talent sont progressivement différenciés selon les premiers choix arbitraires effectués, qui orientent la concentration ultérieure de la demande sur les professionnels ainsi distingués initialement. L'explication des fortes inégalités de revenus artistiques découle ainsi de l'auto-renforcement de la notoriété des vedettes, qui concentrent la demande par ce biais.

Cette préférence au conformisme indépendamment d'une appréciation des différences de talent a été confirmée par quelques tests pratiqués par Chung et Cox. L'hypothèse selon laquelle la distribution du nombre de disques d'or par interprète de musique populaire, aux USA de 1958 à 1969, ait suivi un processus aléatoire ne peut être écartée, tout comme pour la distribution des vedettes de cinéma dans les rôles principaux aux USA ${ }^{21}$. Le processus d'auto-renforcement des avantages initiaux de notoriété par le biais du bouche à oreille est attesté par l'expérience menée par Sagalnik, Dodds et Watt ${ }^{22}$ avec une plateforme de téléchargement de 48 œuvres musicales encore quasiment inconnues. Les expérimentateurs ont séparé les sujets en deux groupes : les personnes du groupe ayant eu connaissance du nombre de téléchargements déjà réalisés dans le passé par les consommateurs ont été orientées dans leurs choix par ces informations, ce qui a accentué les inégalités au profit des chansons déjà les plus partagées. Comme dans l'analogie de Keynes entre le fonctionnement des marchés financiers et celui du concours de beauté dans les journaux londoniens de son époque, où le prix est accordé aux lecteurs dont le choix des six plus jolis visages parmi une centaine de photos se rapproche le plus du classement moyen ${ }^{23}$, l'opinion majoritaire se forme à partir de multiples interactions pour anticiper les comportements des autres.

11 Les variations de cotation des artistes et des professionnels technico-artistiques sont ainsi amplifiées par ce jeu de spéculations. Celui-ci affecte les décisions de programmation. Lors d'un entretien il y a une vingtaine d'années, le directeur d'une scène nationale francilienne m'indiquait ainsi se fier à la rumeur professionnelle pour ses choix de programmation théatrale sans prendre le risque de s'ennuyer en assistant à des spectacles. Les programmateurs doivent aussi entretenir une croyance dans leur capacité à repérer de nouveaux talents pour défendre leur niveau de réputation. Comme il est 
moins risqué de suivre les avis majoritaires que de s'engager seul dans l'accompagnement d'un artiste, des vagues spéculatives promeuvent chaque année les jeunes talents à la mode du moment ${ }^{24}$. Ceux-ci peuvent être délaissés lors de la création suivante si leur ligne esthétique n'est pas jugée suffisamment renouvelée ou si leurs expérimentations artistiques s'éloignent trop des attentes projetées par les programmateurs sur eux à la suite du spectacle distingué.

\section{Conventions et logiques réticulaires de production}

\section{La pluralité des conventions} inné et/ou appris des artistes, et des points de vue constructivistes, axés sur la détermination des cotations des artistes par une contagion mimétique d'opinions, peut être dépassée par une approche conventionnaliste ${ }^{25}$. La mise en équivalence des personnes pour pouvoir mesurer leur état de grandeur dans un monde commun repose sur un principe commun spécifique. La grandeur de ce monde est incarnée par des catégories d'objets et de dispositifs, d'êtres humains exemplaires et de relations harmonieuses entre les êtres, L'équité nécessaire à la viabilité de chaque monde requiert une adéquation entre les efforts individuels déployés pour gagner en grandeur et les épreuves d'attestation de la valeur. Par exemple, la «formule d'investissement » pour gagner en grandeur dans le monde connexionniste est la flexibilité pour s'adapter à de nouveaux projets. Ce monde sera jugé équitable par les personnes si le sacrifice pour développer leur flexibilité est reconnu à sa juste hauteur par des épreuves de réalité.

La pluralité des mondes suscite des frictions entre leurs logiques d'action et d'évaluation quand elles entrent en imbrication. L'accent mis sur le talent en fonction de la singularité des personnes est en résonance avec le monde de l'inspiration tandis que la vision de l'auto-renforcement des réputations découle du monde de l'opinion. La logique d'action et d'évaluation de la libre inspiration entre en tension avec celle de la renommée, ainsi que celle des réseaux selon l'étendue de la capacité connexionniste des artistes et des compagnies pour le financement de leurs projets et la diffusion des spectacles auprès des établissements culturels. Les frictions proviennent aussi de la mise en relation avec la logique d'action et d'évaluation du marché quant à la captation d'un consentement à payer de la part des consommateurs, celle de l'industrie pour la mesure comparative des performances des artistes, celle du monde civique pour les activités théâtrales et chorégraphiques subventionnées selon leur degré de contribution à l'intérêt général, celle du monde domestique selon la chaîne des interdépendances personnelles instaurée dans les familles artistiques.

\section{Les mondes connexionniste et domestique : quelles coopérations ?}

Dans son aménagement du modèle positiviste de Rosen par une mesure comparative et non plus absolue des talents, le sociologue Pierre-Michel Menger se réfère à une logique connexionniste en estimant que la stratification inégalitaire des réseaux d'engagement des artistes s'appuie sur un principe d'appariement entre des professionnels ayant des niveaux de réputation similaires. Cette logique de recherche d'efficacité dans la construction des distributions artistiques et techniques accentue la valorisation 
inégalitaire de la production artistique ${ }^{26}$. La distribution constituée joue un rôle indéniable dans la capacité d'élargissement du réseau de diffusion des spectacles selon le degré de réputation des artistes et professionnels technico-artistiques accueillis. Par exemple, lors d'une réunion publique il $\mathrm{y}$ a déjà presque vingt ans, le directeur d'une autre scène nationale francilienne a avoué procéder à un premier filtrage du millier de dossiers reçus par an en regardant les noms de la distribution artistique et technique. Le repérage d'un professionnel connu et estimé par le directeur était interprété comme un signal d'intérêt pour un examen plus approfondi; les autres dossiers étaient rejetés. Le pouvoir d'attraction des professionnels peut ainsi être pris en compte dans la construction d'une distribution et justifie des inégalités de rémunération au profit des artistes et techniciens les plus renommés. Dans sa position de Directeur du Théâtre et des Spectacles au ministère de la Culture et de la Communication, Robert Abirached a dénoncé dès les années 1980 une dérive dans le recours au vedettariat dans le monde du théâtre subventionnée ${ }^{27}$.

La fidélisation des relations avec des artistes interprètes, des professionnels technicoartistiques est souvent recherchée par des directeurs artistiques en résonance avec la logique d'action et d'évaluation du monde domestique. Les collaborations répétées permettent de gagner en efficacité lors des répétitions par le partage d'un langage artistique spécifique et la consolidation d'une relation de confiance au sein du groupe est une condition favorisant le dépassement de soi dans la dynamique de création. Certains auteurs metteurs en scène comme Joël Pommerat entendent travailler très régulièrement avec les mêmes interprètes, associés au travail d'écriture des pièces par des improvisations à partir des matériaux d'enquête ou fictionnels collectés et par la mise à l'épreuve sur le plateau des propositions scéniques écrites progressivement. Il en résulte, au sens juridique, une œuvre collective possédée par Joël Pommerat. Celui-ci ajoute au salaire des comédiens une part forfaitaire des droits patrimoniaux liés à l'exploitation des œuvres ainsi créées.

Selon une logique d'économie solidaire, la construction de réseaux communautaires sur la base d'affinités esthétiques et éthiques peut inciter à des coopérations interindividuelles ou inter-organisationnelles en s'appuyant notamment sur le partage de ressources non monétaires (locaux, matériel, compétences). La circulation des artistes et des professionnels technico-artistiques peut alors se circonscrire dans une certaine mesure aux organisations partageant les mêmes valeurs. Le directeur artistique d'une compagnie peut par exemple être engagé comme comédien ou danseur sur le projet d'une autre troupe qui partage le local de répétitions. Le développement des collectifs s'appuie aussi sur cette logique de création d'une communauté de travail, avec un équilibre délicat à trouver entre la distribution des ressources financières entre les différents porteurs de projet selon la maturation artistique et la viabilité budgétaire des propositions de création, l'implication des membres dans les projets communs, et une préservation de leurs conditions de vie personnelle.

\section{Le monde civique : quelles relations entre art et action culturelle?}

17 La permanence artistique est également mise en avant comme un moyen d'assurer au mieux les responsabilités territoriale et sociale de la Charte des missions du service public éditée en 1998. Les dispositifs d'action culturelle, comme l'encadrement d'ateliers de pratique artistique, les rencontres en amont ou en aval des représentations, ont pour 
objectif de familiariser les personnes éloignées de la fréquentation des établissements culturels avec les œuvres exigeantes qui y sont programmées. Une présence plus régulière des artistes accroît la disponibilité pour la prise en charge de ces actions pédagogiques et favorise la création de liens de connaissance avec des habitants, susceptibles de se rapprocher du monde des arts de la scène subventionnés ${ }^{28}$. Les compagnies se différencient par leur mode d'organisation du travail d'action culturelle. Certaines d'entre elles introduisent une hiérarchie entre les artistes spécialisés dans l'animation de ces dispositifs et ceux qui sont distribués dans les spectacles. Dans d'autres cas, la participation à ces dispositifs est intégrée à la charge de travail des interprètes, notamment quand la philosophie d'action culturelle des directeurs artistiques considère les interactions avec les univers de non professionnels comme une source d'enrichissement humain et artistique. Une pluralité des fonctions est requise pour le développement des compagnies, en mobilisant les compétences artistiques, pédagogiques, relationnelles avec les collectivités publiques, les partenaires ${ }^{29}$. La fonction de mise en scène est néanmoins plus valorisante que les missions en action culturelle, plutôt chronophages et assez peu rémunérées.

18 La logique d'action et d'évaluation du monde civique, sous-jacente à la mission de service public culturel, a évolué dans une certaine mesure quant aux relations entre les professionnels et les amateurs. La volonté politique de consolider un État-nation culturellement homogène par l'exposition du plus grand nombre aux chefs d'œuvre du patrimoine artistique, prégnante sous le mandat ministériel d'André Malraux entre 1959 et 1969, a cédé du terrain à un projet de développement culturel. Celui-ci est fondé sur une prise en considération de la diversité culturelle sur les territoires d'implantation des organisations artistiques. Alors que les amateurs étaient considérés intrinsèquement comme trop médiocres pour être intégrés dans la programmation des Maisons de la Culture dans les années $1960^{30}$, des metteurs en scène et chorégraphes marquent plus souvent, depuis les années 1990-2000, un intérêt pour l'intégration d'amateurs dans leur distribution. La configuration la plus fréquente consiste à introduire un chœur citoyen, en écho avec la vision idéalisée de la démocratie portée par l'agora athénienne. Un effet de masse peut être recherché pour porter les propos politiques de la pièce comme dans le cas de la mise en scène d'Une saison au Congo de Césaire par Christian Schiaretti ${ }^{31}$ ou de la chorégraphie de Polices! par Rachid Ouramdane ${ }^{32}$. La volonté de se focaliser sur un théâtre de lutte politique est plus rare comme dans le spectacle 81, avenue Victor Hugo, commandé par le Théâtre de la Commune d'Aubervilliers à Olivier Coulon-Jablonka, Barbara MétaisChastagnier et Camille Plagné, qui se fonde sur les témoignages directs de migrants sans papier $^{33}$.

\section{Distributions des compagnies et rapports de diffusion}

\section{La réduction de la taille moyenne des distributions}

L'enquête sur les territoires et ressources des compagnies du spectacle vivant non musical en France ${ }^{34}$ a permis de dégager une typologie géopolitique plus discriminante que les différences habituellement utilisées dans les études déjà parues selon le niveau de budget, la composition de la direction artistique et entre les genres artistiques. Il s'agit du centre de gravité territorial de la diffusion des spectacles. La typologie différencie quatre configurations : les compagnies régionales qui ne diffusent leurs spectacles que dans la 
région de leur siège social; les compagnies transrégionales qui classent leur région au premier rang tout en explorant l'espace national et international; les compagnies multirégionales qui situent l'espace national ou international au premier rang et la région de leur siège social au troisième rang ; les compagnies excentrées qui se focalisent sur les espaces nationaux et internationaux.

Tableau - Évolutions moyennes du volume, des recettes et de la masse salariale par représentation selon les profils de diffusion entre 2007 et 2009 (taux de variation en \%)

\begin{tabular}{|l|l|l|l|l|}
\hline Profil de de & $\begin{array}{l}\text { Nombre par } \\
\text { représentations }\end{array}$ & $\begin{array}{l}\text { Nombre } \\
\text { représen-tations } \\
\text { par spectacle }\end{array}$ & $\begin{array}{l}\text { Recettes } \\
\text { représentation } \\
\text { par représentation }\end{array}$ \\
\hline Régional & 7,2 & $-16,8$ & $-10,6$ & -13 \\
\hline Transrégional & 64,5 & 55,2 & -46 & $-16,5$ \\
\hline Multirégional & 14,4 & $-4,2$ & $-49,7$ & $-19,5$ \\
\hline Excentré & 192,6 & 124,3 & $-46,4$ & $-44,5$ \\
\hline Moyenne & 47,1 & 20,4 & -39 & $-22,2$ \\
\hline
\end{tabular}

Le tableau est construit à partir des donnés des bilans d'activité, des comptes de résultats et des DADS sur les salaires pour les 39 compagnies de l'échantillon qualitatif ayant eu une activité de diffusion continue entre 2007 et 2009. La crise de diffusion se manifeste par la baisse sensible des recettes moyennes par représentation de $39 \%$ en deux ans. Le recul de la masse salariale artistique et technique par représentation semble expliquer un peu plus de la moitié de cette réduction du chiffre d'affaires moyen.

Le salaire journalier moyen des artistes de spectacles en euros constants n'a baissé que de $10 \%$ en moyenne dans le spectacle vivant en 10 ans, entre 2000 et $2009^{35}$. De plus, les entretiens menés dans l'enquête ont indiqué de façon récurrente une résistance à la réduction des salaires distribués aux collaborateurs et une préférence pour la contraction de la marge entre le prix de cession et le coût du plateau. Par conséquent, l'essentiel de cette réduction de la masse salariale par représentation provient d'une diminution de la taille moyenne des distributions artistiques et techniques. Les compagnies se sont adaptées à une demande accrue de petites formes de la part des établissements culturels, qui ont été touchés par une baisse de leur marge disponible pour l'artistique à la suite d'une évolution des subventions inférieure à la hausse des coûts de fonctionnement. Cela réduit les possibilités de financement des dépenses artistiques par la subvention de fonctionnement et oblige les établissements à développer leurs ressources propres.

Cette réduction de la taille moyenne des distributions est un phénomène généralisé. Dans leur actualisation de l'examen de la dite «maladie des coûts» de Baumol et Bowen ${ }^{36}$, William et Hilda Baumol ont mis en avant une contraction de la distribution moyenne dans les pièces de Broadway de 15,6 comédiens en 1946-1947 à 8,1 en 1978-197937. La diminution de la taille moyenne des distributions est associée à un "déficit de qualité " par William et Hilda Baumol. Cette inférence repose sur une hypothèse essentialiste qui considère la quantité de travail artistique comme le seul déterminant de la qualité des 
spectacles. Ce phénomène témoigne néanmoins d'une limitation dans les choix de mise en scène ou de chorégraphie.

\section{Une dégradation des termes de l'échange inégale selon les profils des compagnies} est imputable aussi à une réduction des gains par représentation, liée à la transformation de contrats de cession en contrats de coréalisation, à la négociation de prix de cession plus proches du coût du plateau, ce qui réduit les possibilités d'amortir les frais de montage du spectacle pour la compagnie. Des représentations ont pu aussi être données gracieusement dans le cadre des dispositifs d'action culturelle mis en œuvre notamment dans le cadre d'un développement des spectacles hors les murs au sein des communes. Cette dégradation des termes de l'échange a été confirmée par un récent rapport de l'ONDA, sous la direction de Frédérique Payn, sur les conditions de production et de diffusion de 29 compagnies excentrées subventionnées par le MCC. La forte pression sur les prix de cession est pointée par les répondants ${ }^{38}$.

Larticulation entre la baisse des recettes moyennes et celle de la masse salariale artistique et technique par représentation est différenciée selon les profils de diffusion des compagnies. Le phénomène de contraction de la taille moyenne des distributions concerne le plus les troupes excentrées, pour lesquelles la contraction de la masse salariale artistique et technique par représentation de $44,5 \%$ est proche de celle des recettes moyennes par représentation. Ce profil a connu l'essor le plus important pour la diffusion de leurs spectacles. Les compagnies régionales se spécifient par la baisse la moins importante des salaires artistiques et techniques par représentation $(-13 \%)$ mais celle-ci dépasse la diminution des recettes moyennes par représentation (-10,6\%). On peut en déduire que la contraction du cycle de diffusion des spectacles, avec une baisse estimée de $16,8 \%$ du nombre de représentations par spectacle, a eu pour effet de réduire les salaires distribués par la compagnie et d'aggraver la sous-rémunération du noyau dur des compagnies par rapport aux qualifications et à l'énergie déployées par ses membres. Les compagnies transrégionales et multinationales ont connu des évolutions assez voisines dans les termes de l'échange avec une contraction des recettes moyennes par représentation aussi forte que celle des troupes excentrées mais liées seulement pour $40 \%$ à la baisse de la masse salariale artistique et technique par représentation. Les deux profils se différencient néanmoins par l'évolution contrastée du cycle de diffusion moyen de leurs spectacles.

La capacité au rayonnement national et international dépend de l'entretien d'un répertoire par la compagnie avec des distributions engagées dans les créations de nouveaux spectacles et les reprises ${ }^{39}$. La multiplication des engagements est rendue nécessaire pour s'assurer du renouvellement des droits de tirage de l'assurance chômage des intermittents du spectacle en raison de la baisse de la durée moyenne des contrats et de conditions d'accès à ce régime plus limitées à la suite de la réforme de 2003, dont les orientations ont été globalement confirmées par les protocoles en vigueur depuis 2007. Comme les tournées de spectacles sont discontinues, un problème de disponibilité des artistes et professionnels technico-artistiques de la distribution initiale se pose de façon récurrente. Le coût salarial est alourdi par des répétitions plus longues afin d'intégrer le (s) remplaçant(s) dans la distribution. Cela peut conduire des compagnies à annuler des 
projets de tournée. Des petites aides à la reprise comme celles que pratique l'OARA (Office Artistique de la Région Aquitaine) sont appropriées pour permettre la viabilité de certaines tournées de spectacles.

27 Les distributions associant des chœurs citoyens les renouvellent habituellement à chaque étape de la tournée du spectacle quand le travail artistique demandé reste assez simple. En effet, le prix de cession négocié ne permet pas en général la prise en charge des frais de mission d'un nombre élevé de personnes. Dans le cas de la mise en scène d'Une saison au Congo de Christian Schiaretti, l'implication des personnes recrutées dans le chœur a permis de donner un rôle artistique important à leur présence scénique. Leur participation à la tournée du spectacle prévue au Théâtre des Gémeaux de Sceaux n'a été rendue possible que par leur débrouillardise pour trouver des solutions d'hébergement auprès de leurs familles ou amis en région parisienne. Le choix de Rachid Ouramdane a été de rémunérer les participants du chœur de Polices! avec des cachets d'artiste interprète et non pas de figurant. Le coût de la représentation, autour de 40000 euros, a créé un obstacle rédhibitoire à la tournée du spectacle en dehors des établissements coproducteurs.

\section{Conclusion}

Au-delà de la logique d'action et d'évaluation de l'inspiration, tournée vers la singularisation poétique, les choix de distribution sont donc imbriqués avec une pluralité de principes pour la mise en équivalence du travail artistique afin de mesurer sa valeur. Celle-ci détermine la valeur d'échange des spectacles ainsi que la cotation des rémunérations des artistes et des professionnels technico-artistiques. Dans le monde des arts de la scène subventionnés, une préoccupation majeure provient de la perspective d'un désengagement budgétaire des collectivités territoriales, liée à la baisse de la dotation globale de fonctionnement étatique prévue entre 2015 et 2017, non compensable par un engagement étatique supplémentaire en dehors des bonifications proposées dans les volets culturels des contrats État-Région. Or, selon les observations effectuées entre 1992 et 2002, dans le cas des organisations artistiques soutenues à la fois par l'Etat et les collectivités territoriales, la distribution s'est révélée moins inégalitaire pour les subventions publiques de l'État que pour celles des collectivités locales ${ }^{40}$. Le développement de relations de coopération, portées par les collaborations réticulaires, constitue ainsi un enjeu pour contrecarrer les effets destructeurs et amplificateurs des inégalités de rémunération de la concurrence marchande.

\section{BIBLIOGRAPHIE}

ABIRACHED, Robert, Le Théâtre et le Prince. I. L'embellie 1981-1991, Arles, Actes Sud, 2005.

ADLER, Moshe, «Stardom and talent », The American Economic Review, vol. 75, n 1, 1985, p. 208-212. 
BAUMOL, Hilda, BAUMOL, William J., "The Future of the Theatre and the Cost Disease of the Arts ", Journal of Cultural Economics, vol. 9, n 1, 1985, p. 7-31.

BOLTANSKI, Luc, CHIAPELLO, Ève, Le nouvel Esprit du capitalisme, Paris, Gallimard, 1999.

BOLTANSKI, Luc, THEVENOT, Laurent, Les Économies de la grandeur, Paris, Gallimard, 1991.

CARRÉ, Alice, « Par-delà le mirage », Agôn [En ligne], Points de vue, mis à jour le : 21/05/2015, URL : http://agon.revues.org/3173.

CHARPIN, Jean-Michel et al., Rapport sur le bilan du plan de professionnalisation du secteur du spectacle vivant et enregistré, Paris, IGF-IGAS-IGAC 2008, annexe 1, p. 15.

CORSANI, Antonella, LAZZARATO, Maurizio, Intermittents et Précaires, Paris, Amsterdam, 2008.

COULANGEON, Philippe, « Les musiciens-interprètes », Développement culturel, n 140, juin 2003.

CHUNG, Kee H., cox, Raymond A. K., « Consumer Behavior and Superstardom », Journal of Socio-

Economics, vol. 27, n² 2, 1998, p. 263-270.

CHUNG, Kee H., cox, Raymond A. K., « A stochastic Model of Superstardom: an Application of Yule Distribution ", Review of Economics and Statistics, vol. 76, n 4, 1994, p. 771-775.

GOUYON, Marie, PATUREAU, Frédérique, «Vingt ans d'évolution de l'emploi dans les professions culturelles. 1991-2011», Cultures Chiffres 2014-6, 2014.

GOUYON, Marie, PATUREAu Frédérique, « Tendances de l'emploi dans le spectacle », Cultures Chiffres 2014-2, 2014.

HAMLEN, William A., « Variety and Superstardom », Economic Inquiry, vol. 32, n 3, 1994, p. 395-406. KEYNES, John M., Théorie générale de l'emploi, de l'intérêt et de la monnaie, traduit de l'anglais par Jean de Largentaye, Paris, Payot, 1977, 1 ère éd. 1936.

MAC DONnaLD, Glenn A., " The Economics of Rising Arts ", The American Economic Review, vol. 78, $\mathrm{n}$ $\circ 1,1988$, p. 155-167.

MARSHALL, Alfred, Principles of Economy, London, Mac Millan Company Limited, 1961, $1^{\text {st }}$ ed. 1890. MAIRESSE, François, RochelANDET, Fabrice, Économie des arts et de la culture, Paris, Armand Colin, 2015.

MENGER, Pierre-Michel, Profession artiste. Extension du domaine de la création, Paris, Textuel, 2005.

MENGER, Pierre-Michel, La Profession de comédien. Formations, activités et carrières dans la démultiplication de soi, Paris, DEPS-MCC, 1997.

PAYN, Frédérique, Les Pratiques de production et de diffusion des spectacles des compagnies subventionnées, Paris, ONDA, 2014.

PROUST, Serge, « La pluriactivité dans une économie administrée : le théâtre public », in BUREAU, Marie-Christine, PERRENOUD, Marc, et SHAPIRO, Roberta (dir.), L'Artiste pluriel. Démultiplier l'activité pour vivre de son art, Lille, PU Septentrion, 2009, p. 95-107.

RANNOU, Janine, ROHARIK Ionela, Les Danseurs. Un métier d'engagement, Paris, La Documentation, 2006.

ROSEN, Sherwin, «The Economics of Superstars », The American Economic Review, vol. 71, n 5, 1981, p. 845-858. 
SAGALNIK, Matthew J., DOodS, Peter S., WATTS, Duncan J., « Experimental Study of Inequality and Unpredictability In an Artificial Cultural Market », Science, 311, 2006, p. 854-856.

SMITH, Adam, An Inquiry into the Nature and Causes of the Wealth of Nations, New York, The Modern Library, 1937, 1st ed. 1776.

URFALINO, Philippe, L'Invention de la politique culturelle, Paris, La Documentation française, 1996. URRUTIAGUER, Daniel, « Programming Strategies and Demand in the Performing Arts: the Case of the Forum in Le Blanc-Mesnil, France », International Journal of Arts Management, vol. 17, n 1, 2014, p. 31-42.

URRUTIAGUER, Daniel, Les Mondes du théâtre. Désenchantement politique et économie des conventions, Paris, L'Harmattan, 2014.

URRUTIAGUER, Daniel, « Reprises de spectacles et valorisation de la production artistique », Agôn

[En ligne], Dossiers, (2013) $\mathrm{N}^{\circ} 6$ : La Reprise, Renouveler le contexte, questionner l'institution, mis à jour le : 26/03/2014, URL : http://agon.revues.org/2738.

URRUTIAGUER, Daniel, Guide des études théâtrales. Les professions du spectacle vivant entre les logiques du marché et du service public, Paris, Armand Colin, 2012.

URRUTIAGUER, Daniel, Économie et droit du spectacle vivant en France, Paris, PSN, 2010.

URRUTIAGUER, Daniel, HENRY, Philippe, DUCHENE, Cyril, « Territoires et ressources des compagnies en France », Cultures Études 2012-1, 2012.

URRUTIAGUER, Daniel, « French Decentralisation of the Performing Arts and Regional Economic Disparities ", Journal of Cultural Economics, vol. 29, n 4, 2005, p. 299-312.

\section{NOTES}

1. Marie, Gouyon, Frédérique, Patureau, «Tendances de l'emploi dans le spectacle », Cultures Chiffres 2014-2, 2014, p. 2.

2. Janine, Rannou, Ionela, Roharik, Les Danseurs. Un métier d'engagement, Paris, La Documentation, 2006, p. 19.

3. Philippe, Coulangeon, «Les musiciens-interprètes ", Développement culturel, n 140, juin 2003, p. 1.

4. Marie, Gouyon, Frédérique, Patureau, «Vingt ans d'évolution de l'emploi dans les professions culturelles. 1991-2011 », Cultures Chiffres, 2014-6, 2014, p. 3.

5. Daniel, Urrutiaguer, Guide des études théâtrales. Les Professions du spectacle vivant entre les logiques du marché et du service public, Paris, Armand Colin, 2012, p. 44.

6. Ibid., p. 11.

7. Jean-Michel, Charpin et al., Rapport sur le bilan du plan de professionnalisation du secteur du spectacle vivant et enregistré, Paris, IGF-IGAS-IGAC, 2008, annexe 1, p. 17.

8. La durée moyenne d'un contrat s'est réduite entre 1990 et 2009 de 10 jours à 3 jours pour les artistes et de 14 jours à 6 jours pour les cadres, techniciens et ouvriers intermittents du spectacle (Marie, Gouyon, Frédérique, Patureau, "Tendances de l'emploi dans le spectacle», Cultures Chiffres, 2014-2, 2014, p. 4).

9. Ibid., p.6.

10. Pierre-Michel, Menger, La Profession de comédien. Formations, activités et carrières dans la démultiplication de soi, Paris, DEPS-MCC, 1997, p. 178.

11. Janine, Rannou, Ionela, Roharik, Les Danseurs. Un métier d'engagement, op. cit., p, 173. 
12. Antonella, Corsani, Maurizio, Lazzarato, Intermittents et Précaires, Paris, Amsterdam, 2008, p. 85.

13. L'approche en économie des conventions consiste à comprendre la pluralité des modes de coordination des acteurs en identifiant le principe supérieur commun de référence qui ordonne la grandeur relative des personnes dans chacun des mondes. L'accès à un état supérieur suppose un sacrifice individuel ; la justice de l'ordre requiert que les efforts individuels bénéficient à tous, y compris les plus petits; les états de grandeur ne sont pas attachés aux personnes et dépendent des épreuves de réalité face aux situations rencontrées. Le marché est ainsi appréhendé comme un mode de coordination et non pas comme le seul modèle efficient des échanges comme pour les théories économiques orthodoxes. Selon cette approche, le monde du marché est orienté par le principe supérieur commun de la capacité à attirer un consentement à payer de la part des consommateurs et l'épreuve de réalité pour la mise en équivalence des personnes est le prix des biens et services. D'autres logiques d'action et d'évaluation coexistent. Par exemple, le monde industriel repose sur le principe supérieur commun de la satisfaction rationnelle des besoins et l'épreuve de réalité est basée sur l'efficacité technique.

14. Adam Smith, An Inquiry into the Nature and Causes of the Wealth of Nations, New York, The Modern Library, 1937, $1^{\text {st }}$ ed. 1776, p. 108.

15. Alfred, Marshall, Principles of Economy, London, Mac Millan Company Limited, 1961, $1^{\text {st }}$ ed. 1890 , p. 480.

16. Sherwin Rosen, «The Economics of Superstars ", The American Economic Review, vol. 71, $\mathrm{n}^{\circ} 5$, 1981, p. 845-858.

17. Glenn A., Mac Donald, "The Economics of Rising Art ", The American Economic Review, vol. 78, $\mathrm{n}^{\circ} 1,1988$, p. 155-167.

18. William A., Hamlen, «Variety and Superstardom », Economic Inquiry, vol. 32, n 3, 1994, p. 395-406.

19. David, Hume, «De la norme de goût», Les essais esthétiques. $2^{\text {ème }}$ partie: Art et psychologie, traduit de l'anglais par Renée Bouveresse, Paris, Librairie philosophique J. Vrin, 1974, 1 ère édition 1752,p. 79-104.

20. Moshe, Adler, «Stardom and talent», The American Economic Review, vol. 75, n 1, 1985, p. 208-212.

21. Kee H., Chung, Raymond A. K., Cox, «A stochastic Model of Superstardom: an Application of Yule Distribution ", Review of Economics and Statistics, vol. 76, n 4, 1994, p. 771-775; "Consumer Behavior and Superstardom ", Journal of Socio-Economics, vol. 27, n 2, p. 263-270.

22. Matthew J., Sagalnik, Peter S., Doods, Duncan J., Watts, « Experimental Study of Inequality and Unpredictability In an Artificial Cultural Market », Science, 311, 2006, p. 854-856, cité par François, Mairesse, Fabrice, Rochelandet, Économie des arts et de la culture, Paris, Armand Colin, 2015, p. 216.

23. John Maynard, Keynes, Théorie générale de l'emploi, de l'intérêt et de la monnaie, traduit de

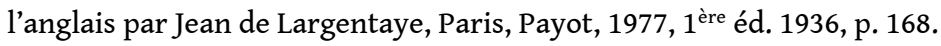

24. Daniel, Urrutiaguer, Économie et droit du spectacle vivant en France, Paris, PSN, 2010, p. 27.

25. Luc, Boltanski, Laurent, Thévenot, Les Économies de la grandeur, Paris, Gallimard, 1991 ; Luc, Boltanski, Eve, Chiapello, Le nouvel Esprit du capitalisme, Paris, Gallimard, 1999; Daniel, Urrutiaguer, Les Mondes du théâtre. Désenchantement politique et économie des conventions, Paris, L'Harmattan, 2014.

26. Pierre-Michel, Menger, Profession artiste. Extension du domaine de la création, Paris, Textuel, 2005, p. 43-45.

27. Robert, Abirached, Le Théâtre et le Prince. I. L'embellie 1981-1991, Arles, Actes Sud, 2005, p. 111-112.

28. Une étude économétrique sur la programmation du Forum, scène conventionnée du BlancMesnil, entre 2002 et 2011 confirme une corrélation positive entre la fréquentation des ateliers et 
celle des spectacles mais à un niveau limité. La faible notoriété des compagnies qui étaient en résidence, sur une période de trois années en principe, explique une fréquentation inférieure à la moyenne pour les spectacles qu'elles ont produits, à l'inverse des spectacles plus " grand public » choisis par les spectateurs lors de l'opération «Faites entrer les artistes ", expérimentée entre 2006 et 2012. L'apport principal des résidences a donc consisté dans l'accompagnement à la structuration administrative et la maturation artistique de ces compagnies (Daniel Urrutiaguer, «Programming Strategies and Demand in the Performing Arts: the Case of the Forum in Le Blanc-Mesnil, France », International Journal of Arts Management, vol. 17, n 1, 2014, p. 31-42).

29. Serge, Proust, «La pluriactivité dans une économie administrée : le théâtre public », in MarieChristine Bureau, Marc, Perrenoud, et Roberta, Shapiro [dir.], L'Artiste pluriel. Démultiplier l'activité pour vivre de son art, Lille, Presses Universitaires du Septentrion, 2009, p. 95-107.

30. Philippe, Urfalino, L'Invention de la politique culturelle, Paris, La Documentation française, 1996, p. 181-183.

31. Entretien de Christian Schiaretti avec Daniel Urrutiaguer, TNP, 16 décembre 2014.

32. Sur un texte de Sonia Chiambretto écrit en 2011. La pièce créée en 2013 a été représentée au Théâtre de la Ville du $1^{\mathrm{er}}$ au 3 avril 2015.

33. Alice, Carré, « Par-delà le mirage ", Agôn [En ligne], Points de vue, mis à jour le : 21/05/2015, URL : http://agon.revues.org/3173.

34. Daniel, Urrutiaguer, Philippe, Henry, Cyril, Duchêne, «Territoires et ressources des compagnies en France », Cultures Etudes 2012-1, 2012.

35. Marie, Gouyon, Frédérique, Patureau, « Vingt ans d'évolution de l'emploi dans les professions culturelles. 1991-2011 », op.cit., p. 4.

36. William J., Baumol, William G., Bowen, "On the Performing Arts: The Anatomy of their Economic Problems ", The American Economic Review, May 1965, vol. 76, n² 2, p. 495-502. Selon cette modélisation, le spectacle vivant est un secteur qui ne peut pas réaliser de gains de productivité et la qualité des représentations est supposée dépendre seulement de la quantité de travail. Comme la hausse des salaires dans les secteurs économiques " progressifs ", qui réalisent des gains de productivité, est supposée se diffuser dans les autres secteurs, il en découle une hausse des prix des spectacles susceptible de réduire la demande. Celle-ci ne peut être contrecarrée selon ce modèle que par le versement des subventions publiques, financées par des transferts financiers des secteurs « progressifs » au détriment de leur compétitivité.

37. Hilda, Baumol, William J., Baumol, «The Future of the Theatre and the Cost Disease of the Arts ", Journal of Cultural Economics, vol. 9, n 1, 1985, p. 7-31.

38. Frédérique, Payn, Les Pratiques de production et de diffusion des spectacles des compagnies subventionnées, Paris, ONDA, 2014, p. 35-38.

39. Daniel, Urrutiaguer, «Reprises de spectacles et valorisation de la production artistique », Agôn [En ligne], Dossiers, (2013) $\mathrm{N}^{\circ} 6$ : La Reprise, Renouveler le contexte, questionner l'institution, mis à jour le : 26/03/2014, URL : http://agon.revues.org/2738

40. Daniel, Urrutiaguer, «French Decentralisation of the Performing Arts and Regional Economic Disparities ", Journal of Cultural Economics, vol. 29, n 4, 2005, p. 299-312. 


\section{RÉSUMÉS}

Les explications économiques usuelles des inégalités de rémunération du travail artistique se partagent entre une focalisation positiviste sur le talent objectivé, dont la rareté attirerait une concentration de la demande, et une vision constructionniste basée sur l'effet boule de neige des engouements collectifs. Selon une perspective conventionnaliste, l'article dégage la pluralité des logiques de production qui orientent les choix de distribution. Il s'agit notamment des appariements selon la renommée, la fidélisation, la recherche d'une solidarité, la vision du rôle civique des artistes. L'exploitation de l'étude sur les territoires et les ressources des compagnies en France apporte des éclairages sur les inégalités de valorisation qui découlent des capacités de diffusion dans un contexte budgétaire plus contraint qu'avant, source d'une réduction importante de la taille moyenne des distributions et de négociations plus serrées sur les prix de cession.

Usually economic explanations of the artists' wage inequalities are balancing from a positivist focus on the objectified talent, for which scarcity should attract most of the demand, and a constructivist view on the information cascades which cause collective enthusiasms. Following a conventionalist view, the article is highlighting the pluralist logics of production which influence the cast choices. Job matching is especially varying according to the artists' renown, the directors' search of loyalty or solidarity, and the view on the artists' civic role. The survey on the territories and resources of the performing arts companies in France gives information on the income inequalities according to the capacities of diffusion in a tighter budgetary situation. With this evolution came an important decrease of the mean size of casts and tougher negotiations on the sales price.

\section{INDEX}

Keywords : convention, diffusion, distribution, network, performing arts, value Mots-clés : convention, diffusion, distribution, réseau, spectacle vivant, valeur 